\title{
ON THE RELATIONS BETWEEN THE DIRECTION OF TWO-DIMENSIONAL ARM MOVEMENTS AND CELL DISCHARGE IN PRIMATE MOTOR CORTEX ${ }^{1}$
}

\author{
APOSTOLOS P. GEORGOPOULOS, ${ }^{2}$ JOHN F. KALASKA, ${ }^{3}$ ROBERTO CAMINITI, ${ }^{4}$ AND JOE T. MASSEY ${ }^{5}$
}

Departments of Physiology and Neuroscience, The Johns Hopkins University School of Medicine, Baltimore, Maryland 21205

Received October 30, 1981; Revised April 30, 1982; Accepted May 21, 1982

\begin{abstract}
The activity of single cells in the motor cortex was recorded while monkeys made arm movements in eight directions (at $45^{\circ}$ intervals) in a two-dimensional apparatus. These movements started from the same point and were of the same amplitude. The activity of 606 cells related to proximal arm movements was examined in the task; 323 of the 606 cells were active in that task and were studied in detail.

The frequency of discharge of 241 of the 323 cells (74.6\%) varied in an orderly fashion with the direction of movement. Discharge was most intense with movements in a preferred direction and was reduced gradually when movements were made in directions farther and farther away from the preferred one. This resulted in a bell-shaped directional tuning curve. These relations were observed for cell discharge during the reaction time, the movement time, and the period that preceded the earliest changes in the electromyographic activity ( $\sim 80 \mathrm{msec}$ before movement onset). In about $75 \%$ of the 241 directionally tuned cells, the frequency of discharge, $D$, was a sinusoidal function of the direction of movement, $\theta: D=b_{0}+b_{1} \sin \theta+b_{2} \cos \theta$, or, in terms of the preferred direction, $\theta_{0}: D=$ $b_{0}+c_{1} \cos \left(\theta-\theta_{0}\right)$, where $b_{0}, b_{1}, b_{2}$, and $c_{1}$ are regression coefficients. Preferred directions differed for different cells so that the tuning curves partially overlapped.

The orderly variation of cell discharge with the direction of movement and the fact that cells related to only one of the eight directions of movement tested were rarely observed indicate that movements in a particular direction are not subserved by motor cortical cells uniquely related to that movement. It is suggested, instead, that a movement trajectory in a desired direction might be generated by the cooperation of cells with overlapping tuning curves. The nature of this hypothetical population code for movement direction remains to be elucidated.
\end{abstract}

The importance of the motor cortex for voluntary limb movements in the primate is well established. It is supported by the results of lesion, electrical stimulation, and single cell recording experiments carried out by many investigators (reviewed in detail by Evarts, 1981). The

\footnotetext{
${ }^{1}$ This research was supported by United States Public Health Service Grants NS17413, EY03167, and NS07226 which we gratefully acknowledge. We thank Drs. Allyn Kimball and Kenneth Johnson for valuable help with the periodic regression

${ }^{2}$ To whom correspondence should be addressed at Department of Neuroscience, The Philip Bard Laboratories of Neurophysiology, 725 North Wolfe Street, The Johns Hopkins University School of Medicine, Baltimore, MD 21205.

${ }^{3}$ Postdoctoral Fellow of the Medical Research Council of Canada, 1978-1981. Present address: Centre de Recherche en sciences neurologiques, Faculté de Medécine, Université de Montréal, Montréal, Québec, H3C 3J7, Canada.

${ }^{4}$ Consiglio Nazionale delle Ricerche-North Atlantic Treaty Organization Fellow, 1980-1981; Howell-Cannon Foreign Scholar, 1981-1982. Present address: Istituto di Fisiologia umana dell 'Universitá, II Cat-
}

role of the motor cortex in motor control and the mechanisms by which this function is carried out are not well understood. Studies of single neurons in behaving monkeys, begun with the pioneer experiments of Jasper (1958) and Evarts (1966), have centered on defining the relations of cell discharge to muscular force and movement parameters (Evarts, 1968, 1969; Humphrey et al., 1970; Schmidt et al., 1975; Smith et al., 1975; Thach, 1978; Hepp-Reymond et al., 1978; Hamada and Kubota, 1979; Cheney and Fetz, 1980); the presence, kind, and importance of peripheral somatic input (Conrad et al., 1975; Lemon and Porter, 1976; Evarts and Fromm, 1977; Wong et al., 1978; Fetz et al., 1980; Wolpaw, 1980); and the

tedra, Facoltá di Medicina e Chirurgia, Cittá Unviersitaria, 00185 Roma, Italy.

${ }^{5}$ William S. Parsons Visiting Professor, 1978-1979. Present address: Applied Physics Laboratory, The Johns Hopkins University, Laurel, MD 20810. 
interactions between peripheral and central events (Evarts and Tanji, 1976; Tanji and Evarts, 1976).

Evarts $(1968,1969)$ studied the activity of single cells in the motor cortex of the monkey during push-pull movements. He observed that $(a)$ a population of cells in the arm area was related to these movements, $(b)$ the changes in neuronal activity were locked temporally to the onset of movement, and $(c)$ these changes were often opposite in sign (increase or decrease of discharge) for the two directions of movement. He also observed that the activity of motor cortical cells was related to the muscular force generated by animal. This finding has been confirmed recently (Hepp-Reymond et al., 1978; Thach, 1978; Cheney and Fetz, 1980). The effect is observed most clearly in cells that probably entertain direct monosynaptic connections to spinal motoneurons (Cheney and Fetz, 1980), and it may operate for different ranges of forces in different cells (Hepp-Reymond et al., 1978). The relative influences on cell activity of these two factors, the direction of movement and muscular force, remain to be determined. Indications that the former might be stronger than the latter (Schmidt et al., 1975) need to be re-examined in the light of the recent observations mentioned above regarding the optimal conditions for the occurrence of the force effect. In a different experiment, it was observed that approximately equal proportions of motor cortical cells were related to muscle activity, hand position, and direction of intended movement (Thach, 1978). The relations of single cells in the motor cortex to other parameters of movement (e.g., velocity) were not found to be strong or consistent (Humphrey et al., 1970; Hamada and Kubota, 1979), although the relation to velocity was improved when the activity of several cells recorded simultaneously was pooled (Humphrey et al., 1970).
Neuronal activity in the primate motor cortex, then, can be related to the force exerted by the animal, to the direction, or to the velocity of the movement, alone or in combination. Of the three, the relations to the direction of the movement have been least well analyzed. In previous studies, two directions of movement have been utilized that were usually opposite (Evarts, 1968; Schmidt et al., 1975; Thach, 1978; Hamada and Kubota, 1979; Fetz et al., 1980); the differences in neuronal discharge observed under these conditions ranged from excitation in one and inhibition in the other ("reciprocal") to various degrees of excitation alone. It is obvious that more than two directions of movement must be examined to determine the relations of single neuron activity to the direction of movement. There are also other important questions in this respect. For example, is a cell activated uniquely in only one direction of movement? If not, what are the quantitative relations between cell activity and direction of movement? Preliminary results of this study were presented elsewhere (Georgopoulos et al., 1980).

\section{Materials and Methods}

Four male rhesus monkeys ( 4 to $5 \mathrm{~kg}$ ) were used. They were trained to move a lightweight frictionless manipulandum over a plane surface and capture lighted targets within a circle attached to the manipulandum in a reaction task (Fig. 1). The plane was tilted $15^{\circ}$ from the horizontal toward the animal's body. With gravity acting on the manipulandum, static forces on the $x$ or $y$ axis of the plane did not exceed $94 \mathrm{gm}$. The two-dimensional apparatus used, the behavioral task, and some aspects of the performance of the animals have been described previously (Georgopoulos et al., 1981). Briefly, a circular pattern of LEDs (light-emitting diodes) was used, with
A.

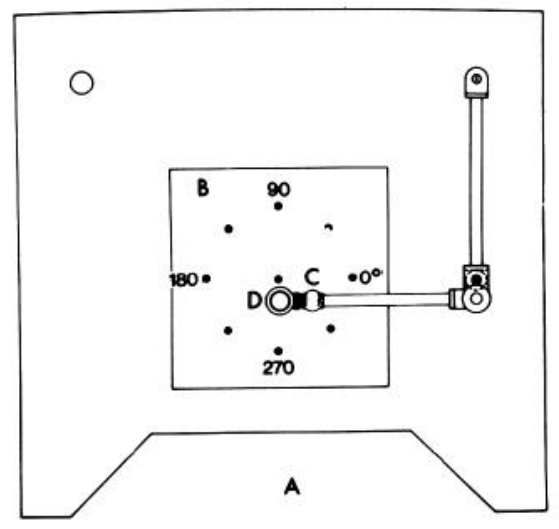

B.

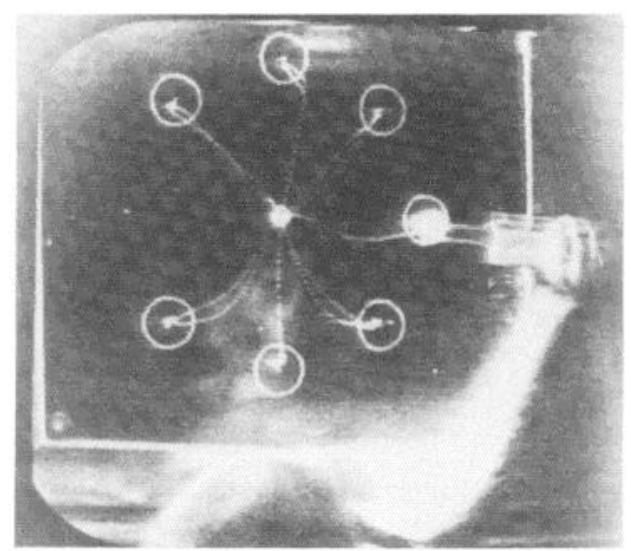

C.

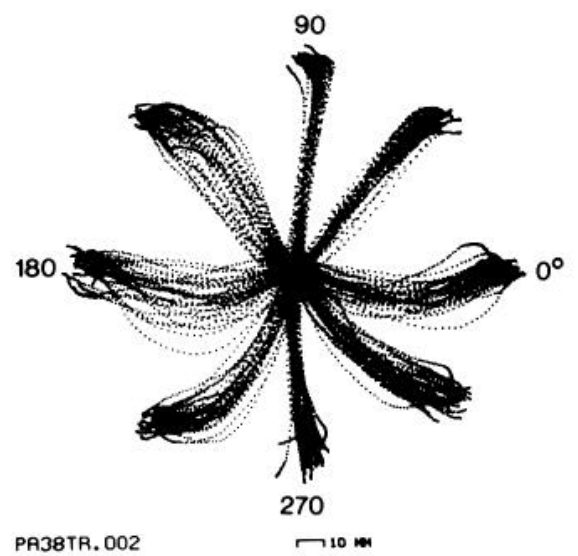

Figure 1. A, Diagram of the behavioral apparatus. The monkey sits at position $A$, facing a 25 -cm square working surface ( $B$ ) on which there are nine light-emitting diodes (LEDs). One LED is at the center of the working surface and eight are on a circle with an $8-\mathrm{cm}$ radius; they are numbered from 0 to $315^{\circ}$ counterclockwise. The monkey grasps an articulated manipulandum at its end $(C)$ and moves it across the $x-y$ surface of the plane to capture within a clear plastic circle $(D)$ whichever LED is illuminated. The plane is tilted $15^{\circ}$ from the horizontal toward the animal. $B$, Overhead view of a monkey performing the task displayed on a television monitor. The monkey has moved the manipulandum from the center to the target LED (in this case, the movement direction is $0^{\circ}$ ) to complete a trial. The trajectories of movement for this trial and for a few previous trials are superimposed on the television image as light lines. The small circles are the 25-mm-diameter target windows around each target LED. The starting (center) window had a diameter of $15 \mathrm{~mm}$. C, Trajectories of 30 movements to each target made by a well trained monkey. Each dot is the position of the center of the target-capturing circle on the end of the manipulandum taken at 10-msec intervals. 
one LED at the center and eight at the circumference of a circle with an $8-\mathrm{cm}$ radius. In a typical trial, the animal first captured the center light and held that position for a variable period of time ( 1 to $3 \mathrm{sec}$ ); then a peripheral LED came on and the animal had to capture it with the freely movable manipulandum to receive a liquid reward. Since the eight targets were arranged equidistantly on the circumference of the circle, the directions of the movement trajectories made from the center to the peripheral targets covered the whole circle at intervals of $45^{\circ}$. No restrictions were imposed on the trajectories of the movements. The 8 targets were presented in a randomized block design (Cochran and Cox, 1957).

Neural studies. After a period of 1 to 2 months of training, a recording chamber with an internal diameter of $18 \mathrm{~mm}$ was placed over the arm area of the motor cortex under general anesthesia and a $\mathrm{T}$ bar was positioned on the skull for the purpose of immobilizing the head during the experiment. Both the chamber and the $\mathrm{T}$ bar were held in place on the skull by dental acrylic. The chamber was closed each day by a tightly fitting plug which was lowered to 2 to $3 \mathrm{~mm}$ above the dura and sealed in place and covered with a cap; the animal was left free in the home cage overnight. On each recording day, the head was immobilized mechanically, the plug was removed from the chamber, and a Chubbuck mechanical microdrive (described in Mountcastle et al., 1975) was attached to the fluid-filled and hydraulically sealed chamber. The drive allowed positioning of the microelectrode for penetration anywhere within a 14mm-diameter circle and forward motion controlled to the $1-\mu \mathrm{m}$ level. The microelectrodes were glass-coated platinum/iridium alloy wires, shaped by etching and selected to optimize small tips for recording, combined with sufficient shaft stiffness to allow penetration of the intact dura. Tip impedances were 2 to 3 megohms. Ordinarily, one microelectrode penetration was performed per day. Its location on the surface of the brain was marked on a grid map of the chamber. Recording times were limited to 6 to $7 \mathrm{hr}$. Recording continued for about 20 days in each hemisphere. An $x$-y electro-oculogram (EOG) was recorded in two animals using implanted $\mathrm{Ag} / \mathrm{AgCl}$ electrodes. The electromyographic (EMG) activity of the following muscles (Howell and Straus, 1933) was sampled during performance in the task using intramuscular, multistranded, stainless steel wires: deltoid íclavicular and spinal parts), trapezius (cranial and caudal parts), pectoralis major, teres major, latissimus dorsi, triceps (lateral and long heads), biceps, brachialis, brachioradialis, neck muscles (deep to cranial trapezius), thoracic paraspinal, and flexors and extensors of the hand. EMG recordings were done separately from neural recording sessions.

The action potentials of single neurons were recorded extracellularly, identified by the criteria of Mountcastle et al. (1969), and displayed on the screen of regular and memory oscilloscopes. They were amplified and processed through head stages, power amplifiers, high rolloff filters, a differential amplitude discriminator, and second stage squelch circuits. Every effort was made to isolate for study the initially negative action potentials of neurons when as small as possible, compatible with reliable isolation, and when accompanying others, both signs that the neuron is still some distance from the electrode tip and thus least likely to be damaged. Once a neuron was thus isolated, a detailed examination of the animal was carried out by at least two observers to determine $(a)$ whether the cell activity was related to the movements of a body part and, if so, to which one and (b) the cell's response to passive manipulations of skin, muscles, joints, and deep tissues with the animal relaxed. Then the cell activity was recorded while the monkey performed in the task. A record was kept of the depth at which each cell was isolated along the entire length of each penetration from the first cells recorded after entry into the cortex until entry into the white matter as judged by the absence of initially negative potentials and the recording of purely positive (fiber) spikes. At the end of some penetrations, small lesions were made to facilitate the reconstruction of the penetration; typically, a $3-\mu \mathrm{A}$ current was passed through the tip of the microelectrode for 3 sec.

At the end of the experiment, penetrations were made in which several lesions were placed for marking purposes. After 2 to 3 days, the animal was killed with an overdose of pentobarbital. The brain was fixed in buffered formalin, embedded in celloidin, and sectioned every $20 \mu \mathrm{m}$, and each section was stained with thionin. Microelectrode penetrations in which lesions were made were reconstructed from these sections. 'The point of entry into the brain of penetrations in which no lesions were made was determined relative to the identified penetrations using the grid map of the chamber.

Data collection. A PDP11/20 laboratory minicomputer was used to control the lights on the plane, to monitor and record behavior, and to collect neural (interspike intervals with a resolution of $0.1 \mathrm{msec}$ ) and analog ( $x, y$ position of the manipulandum of the plane, EOG, and EMG) data. Analog data were sampled at 100/ sec. All data were stored on-line in digital form. Neuronal data were displayed as rasters of spikes upon a Tektronix 613 oscilloscope screen. The trajectories of the movements also were displayed at the same time.

Data analysis. The intensity of cell discharge (impulses per sec) was calculated for four epochs: $(a)$ the control period, during which the animal held the manipulandum aligned at the center of the plane without moving; (b) the reaction time (RT), from the appearance of the peripheral target to the onset of movement, as judged from the velocity record using a threshold cross; (c) the movement time (MT), from the onset of movement to its approximate end (i.e., when the velocity relurned to the value that it had when the beginning of movement was judged to occur); and $(d)$ the total experimental time (TET), from the appearance of the peripheral target to the end of the movement: TET $=\mathrm{RT}+$ MT.

The timing of the first change in cell activity was determined using plots of difference in activity between consecutive 20-msec bins and the mean (per bin) control discharge level; an acceptable change was deemed to occur when at least three consecutive bins showed consistent change (increase or decrease) with at least two of the three exceeding the mean control level by at least $50 \%$. This method was resistant to errors caused by 
isolated high or low bin values, for it incorporated a measure of consistency as well. An example illustrating this method is shown in Figure 2.

Standard statistical (Snedecor and Cochran, 1967) and display techniques were used to analyze the data, including analysis of variance, perievent time histograms, and scatter diagrams. A first degree periodic (sinusoidal) regression was performed on the mean discharge rates as follows. Let $y_{i}(i=1,2,3, \ldots, 8)$ be the mean rate at which a cell discharges during movements at directions $\theta_{i}=0,45,90, \ldots, 315^{\circ}$, respectively. The regression to be fitted is

$$
y=b_{0}+b_{1} \sin \theta+b_{2} \cos \theta
$$

where $b_{0}, b_{1}$, and $b_{2}$ are regression coefficients. The least square unbiased estimators for $b_{0}, b_{1}$, and $b_{2}$ are as follows:

$$
\begin{gathered}
b_{0}=\bar{y}=1 / 8\left(y_{1}+y_{2}+y_{3}+y_{4}+y_{5}+y_{6}+y_{7}+y_{8}\right) \\
b_{1}-1 / 4\left[\frac{1}{\sqrt{2}}\left(y_{2}+y_{4}-y_{6}-y_{8}\right)+\left(y_{3}-y_{7}\right)\right] \\
b_{2}=1 / 4\left[\frac{1}{\sqrt{2}}\left(y_{2}-y_{4}-y_{6}+y_{8}\right)+\left(y_{1}-y_{5}\right)\right]
\end{gathered}
$$

A measure of the goodness of the regression fit is the coefficient of determination, $R^{2}$, which is the proportion of the total variation $\left(\Sigma y^{2}\right)$ that is explained by the
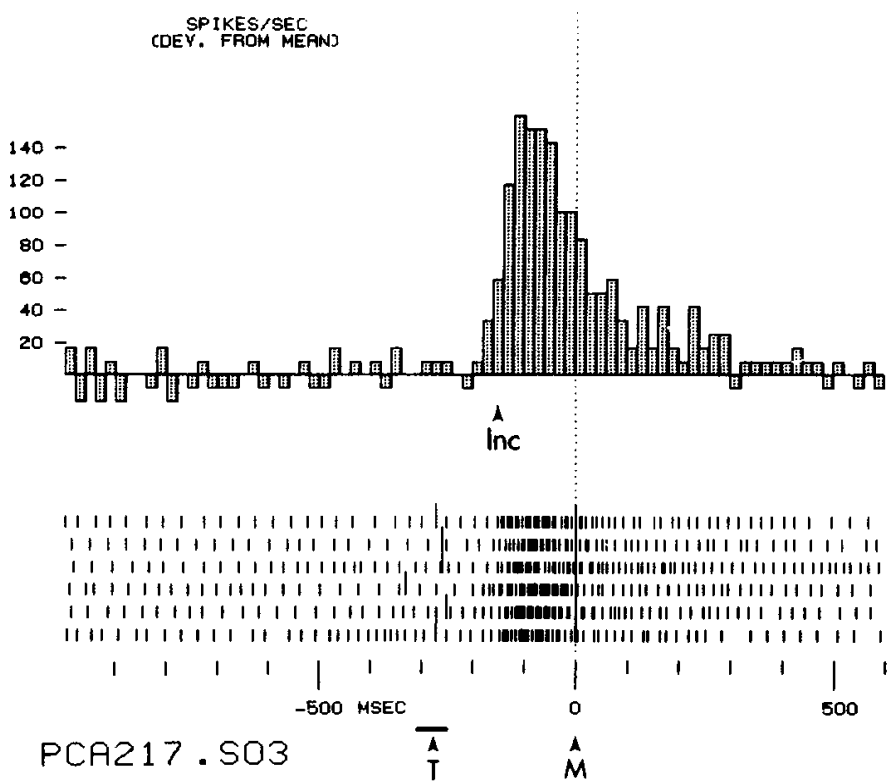

Figure 2. Example of the determination of the timing of the first change in neuronal activity using the method described in the text. Impulse activity was recorded from a single neuron during 6 movements toward the same target and is displayed as a raster (bottom) and as a perievent histogram (top). All trials and the histogram are oriented to the onset of movement. The time of appearance of the target for each trial is indicated by a longer vertical line in the spike train to the left of the orientation point. The histogram is plotted as the impulses per sec deviation from the mean control rate of discharge observed before the appearance of the target LED. The bin width of the histogram is $20 \mathrm{msec}$. Inc, Onset of the initial increase of activity as determined by method described in the text; $M$, onset of movement; $T$, mean time $\pm 1 \mathrm{SD}$ of the appearance of the target. regression $\left(\Sigma \hat{y}^{2}\right)$. Substituting, $\Sigma y^{2}=\sum_{i}\left(y_{i}-\bar{y}\right)^{2}$, and $\Sigma \hat{y}^{2}=4\left(b_{1}^{2}+b_{2}^{2}\right)$, we get

$$
R^{2}=\frac{4\left(b_{1}^{2}+b_{2}^{2}\right)}{\sum(y-\bar{y})^{2}}
$$

Equation 1 can be re-expressed in terms of the peak of the sine wave ("preferred direction") as follows:

$$
y=b_{0}+c_{1} \cos \left(\theta-\theta_{0}\right)
$$

where $\theta_{0}$ is the preferred direction and $c_{1}=\sqrt{b_{1}^{2}+b_{2}^{2}}$. The coefficient $c_{1}$ denotes the increase in discharge over the overall mean $b_{0}$ at the preferred direction $\theta_{0}$. The preferred direction can be calculated using trigonometric moments (see Mardia, 1972, pp. 25-26) or, alternatively, from equation 1 as follows. First calculate $\theta_{0}^{\prime}$ :

$$
\theta_{0}^{\prime}=\tan ^{-1} \frac{b_{1}}{b_{2}} ; \quad-45^{\circ}<\theta_{0}^{\prime}<45^{\circ}
$$

Then, according to the quadrant, $\theta_{0}$ is given by:

$$
\begin{gathered}
\theta_{0}=\theta_{0}^{\prime}, \quad \text { if } b_{1}>0 ; b_{2}>0 \\
\theta_{0}=\theta_{0}^{\prime}+180^{\circ}, \quad \text { if } b_{2}<0 \\
\theta_{0}=\theta_{0}^{\prime}+360^{\circ}, \text { if } b_{1}<0 ; b_{2}>0
\end{gathered}
$$

The proportional increase in discharge at the preferred direction over the overall mean, $b_{0}$ (equation 2 ), could be used as an index, $I$, of the directional modulation:

$$
I=\frac{c_{1}}{b_{0}} ; \quad b_{0}>0
$$

\section{Results}

\section{Behavioral data}

The task in this study required the monkey to make visually guided aimed movements of the arm in different directions away from a common starting position to targets at eight locations on a plane. Before a target appeared, the monkey held the manipulandum over the central LED and scanned the working surface by making spontaneous eye saccades. When the target appeared, the eyes saccaded to foveate it at typical latencies of 150 to $170 \mathrm{msec}$, and then the arm began to move toward the target; the average behavioral reaction time, from the appearance of the target to the onset of arm movement, was $265 \pm 60$ msec (mean $\pm \mathrm{SD} ; n=2500$ movements). Recordings of EMG activity indicated that arm movements in different directions were performed by differential activation of muscles operating on the shoulder joint, shoulder girdle, and proximal arm: deltoid, pectoralis major, trapezius (especially caudal part), teres major, and latissimus dorsi (Massey et al., 1981). The earliest changes in EMG activity occurred most commonly at about $80 \mathrm{msec}$ before the onset of arm movement. These early changes were observed in different muscles for different directions of movement; for example, changes were seen in the pectoralis major for movements at $180^{\circ}$ (right arm) and in the clavicular part of deltoid for movements at $90^{\circ}$.

\section{Data base}

The activity of 606 single motor cortical neurons was 
related to movements of the contralateral arm at the shoulder and/or elbow. Figure 3 shows the point of entry of the microelectrode penetrations $(n=69)$ in which such cells were encountered in five hemispheres (three right and two left) of four monkeys. Of these 69 penetrations, $49(71 \%)$ were identified histologically. All 69 penetrations were within the proximal arm area of the motor cortex as outlined by Woolsey et al. (1950) (Fig. 3, left bottom), which includes area 4 and a posterior strip of area 6 (Wiesendanger, 1981). No penetrations were made in the bank of the arcuate sulcus.

All 606 arm-related cells were tested in the task; 283 of the $606(47 \%)$ were not active under the task circumstances but discharged during other arm movements (e.g., during retraction or elevation of the arm). The

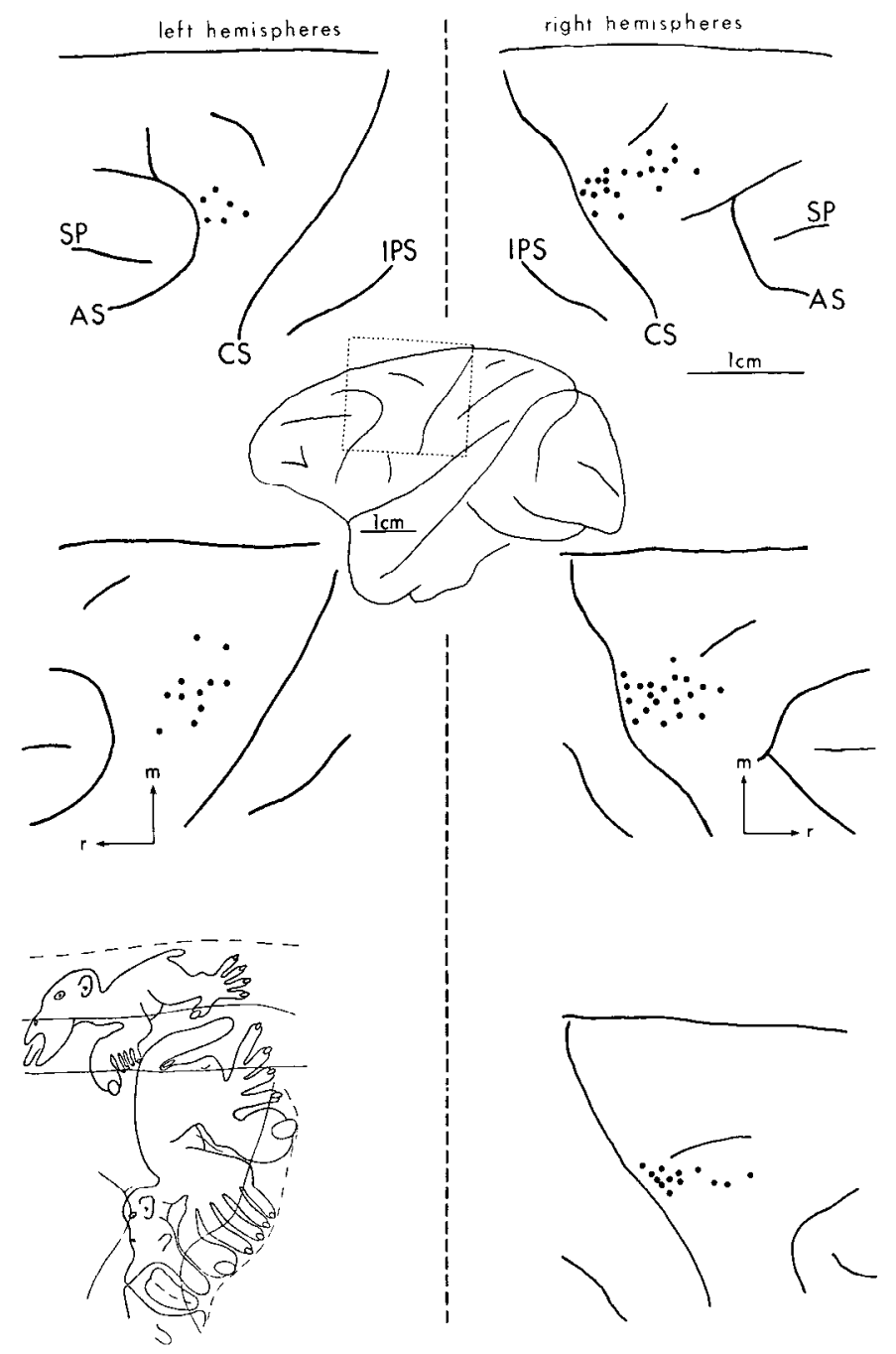

Figure 3. The drawing in the center shows a lateral view of the macaque cerebral hemisphere. The area outlined is enlarged in the five brain figurines to show the frontal region of three right and two left hemispheres where cells related to upper proximal arm movements were encountered in microelectrode penetrations $(n=69)$. On the left bottom, a somatotopic map of the motor (and supplementary motor) cortex is outlined as described by Woolsey et al. (1950). Notice that penetrations with cells related to proximal arm movements in the present study were located at approximately the proximal arm area of the Woolsey map. AS, SP, CS, and IPS are the arcuate, prinicpal, central, and intraparietal sulci, respectively. remaining 323 cells (53\%) were active in the task and form the sample for the quantitalive study described below. Of these, 223 cells $(69 \%)$ were recorded in histologically identified penetrations.

\section{Quantitative studies: Relations to the direction of movement}

Several aspects of cell activity (intensity of discharge, sign of change, and the timing and time course of these changes) frequently varied in an orderly fashion with the direction of movement, alone or in combination. Almost all of the 323 cells studied showed changes in discharge frequency in association with more than one, and commonly with all, directions of movement.

All directional analyses described below refer to the right arm; data obtained when the animal was performing with the left arm (i.e., when recording from the right hemisphere) have been converted to a mirror right arm image. A polar coordinate system was used for labeling the eight directions of movement tested $(0,45,90,135$, $180,225,270$, and $315^{\circ}$; Fig. 1); the 0 to $180^{\circ}$ axis was parallel to the frontal plane, and the 90 to $270^{\circ}$ axis was at the midsagittal plane of the body. Movements at 315, 0 , and $45^{\circ}$ were directed to the ipsilateral (to the arm; contralateral to the hemisphere studied) space; those at 135,180 , and $225^{\circ}$ were directed to contralateral space.

The relations between movement direction and $(\alpha)$ frequency of discharge, $(b)$ kind of change (increase or decrease from the control discharge rate), and (c) onset time of first change in neuronal discharge are discussed separately below. The relations with discharge frequency were analyzed for RT, MT, and TET epochs.

Relations between the frequency of discharge and direction of movement: Directional tuning curve. Significant relations between cell discharge and the direction of movement were observed during the RT (in 232 of 323 , $71.8 \%$ of the cells), the MT (in 286 of $323,88.5 \%$ of the cells), and the TET (in 296 of $323,91.6 \%$ of the cells) (analysis of variance, $F$ test, $p<0.05$ ). Directional relations also were observed before the earliest EMG changes (i.e., before $80 \mathrm{msec}$ preceding the onset of movement). We describe below in detail the results for the TET epoch because, frequently, the neuronal response occupied parts of both the RT and the MT. Similar results were obtained for the other epochs.

A directional preference was present in 241 cells ("directionally tuned cells"). These cells discharged at higher frequencies to one movement direction and at lower rates to other directions. This resulted in a bellshaped directional tuning curve. In many cells, tuning curves were sinusoidal functions of the direction of movement; that is, the frequency of discharge, $D$, varied sinusoidally with the angle, $\theta$, of the direction of movement, according to the equation (see "Materials and Methods")

$$
D=b_{0}+b_{1} \sin +b_{2} \cos \theta
$$

or, equivalently,

$$
D=b_{0}+c_{1} \cos \left(\theta-\theta_{0}\right)
$$

where $\theta_{0}$ is the preferred direction and $b_{0}, b_{1}, b_{2}$, and $c_{1}$ are regression coefficients. These coefficients, and a measure of the goodness of the fit (coefficient of determination, $R^{2}$ ) were calculated using a sinusoidal regres- 
sion analysis (see "Materials and Methods"). Equation 1 described adequately the directional relations of about $75 \%$ of the 241 cells $\left(R^{2} \geq 0.7\right)$; the fit was excellent in $26 \%$ of the cells $\left(R^{2} \geq 0.9\right)$, very good in $26 \%\left(0.9>R^{2}\right.$ $\geq 0.8)$, and good in $23 \%\left(0.8>R^{2} \geq 0.7\right)$. It was not as good $\left(R^{2} \leq 0.7\right)$ in 60 of the 241 cells $(25 \%)$; these cells did possess bell-shaped tuning curves, but their responses were limited to a few movement directions. However, only 1 cell discharged in only one of the eight directions of movement tested.

Figure 4 illustrates the sinusoidal relation between cell discharge and the direction of movement. On the upper half, the spike trains of five replications are shown for each direction of movement (indicated by the arrows in the center). The frequency of discharge of this cell varied sinusoidally with the direction of movement. This is illustrated in the lower half of Figure 4, in which the mean discharge rates during TET are plotted as a function of the direction of movement. The tuning curve was fitted using regression equation 1 above, which was for this cell, $D=32.37+7.281 \sin \theta-21.343 \cos \theta\left(R^{2}=\right.$ 0.946 ) or, equivalently, $D=32.37+22.55 \cos \left(\theta+\theta_{0}\right)$. The preferred direction $\left(\theta_{0}\right)$ for this cell was at $161^{\circ}$, and the index of directional modulation (see "Materials and Methods") was 0.7 .

Preferred direction and directional modulation differed for different cells. The distribution of preferred directions is shown in Figure 5. It can be seen that there was a higher percentage of cells with preferred directions at $45^{\circ}$ (50 of $241,20.7 \%$ of the cells) than at $225^{\circ}$ (15 of $241,6.2 \%$ of the cells) with intermediate percentages in between. Similar distributions were observed in the other epochs (RT and MT). The average directional modulation index was $0.837 \pm 0.398$ (mean $\pm \mathrm{SD}$ ) for $\mathrm{TEl}, 0.911$ \pm 0.454 for $\mathrm{RT}$, and $0.925 \pm 443$ for MT; that is, maximum increase (or decrease) was, on the average, $84 \%, 91 \%$, and $93 \%$ of the overall mean discharge rate observed during TET, RT, and MT, respectively. These mean discharge rates ( $b_{0}$ in equations 1 and 7 above) were approximately 15 impulses/sec for each of the three epochs above (range, 1.6 to 58 ).

The results described above dealt with the directional characteristics of the 241 cells that possessed directional preference out of 296 cells that showed significant relations to the direction of movement. The remaining 55 cells $(18.6 \%)$ did not seem to possess a preferred direction because they discharged to different directions of movement in an irregular fashion.

Relations between movement direction and the kind of first change in neuronal discharge. The kind of first change in neuronal discharge (increase or decrease from the control rate) was a function of the direction of movement. An increase in activity occurred most frequently when the movement direction was at or near $\left( \pm 22.5^{\circ}\right)$ the cell's preferred direction and less frequently the farther away the movement direction was from that direction. The opposite was observed for decrease and no change in discharge. These are illustrated in Figure 6.

Onset times of first changes in neuronal discharge. The time of onset of the first change in neuronal discharge often varied with the direction of movement. An increase in cell discharge tended to occur earlier the closer the movement direction was to the cell's preferred direction. For example, when the direction of movement was at or within $\pm 22.5^{\circ}$ of the cell's preferred direction, 216 of 241 cells $(90 \%)$ showed an increase in activity as a first change, a percentage in accord with Figure 6 . The distribution of the onset times of the increase in discharge among these 216 cells is shown in Figure 7 . It can be seen that 189 of 216 cells $(87.5 \%)$ were activated before the onset of movement. This percentage decreased to $80.2 \%$, $66 \%$, and $64 \%$ when the movement direction and the cell's preferred direction differed by 45,90 , and $135^{\circ}$, respectively; in addition, in these cases, fewer and fewer cells were activated, overall, as indicated in Figure 6. The proportion of cells activated before the earliest EMG changes showed a similar trend. The onset times of decrease in cell discharge followed an opposite pattern: decrease occurred earlier the farther the movement direction was from the cell's preferred direction.

Directional composition of the "early" neuronal population. The results of the two preceding sections suggest that, when a movement in a particular direction is to be made, $(a)$ cells with preferred directions at or near the direction of the upcoming movement will be activated and $(b)$ cells with preferred directions opposite or far from the direction of movement will be inhibited. This was tested by analyzing the directional properties of cells engaged early in the reaction time (i.e., cells whose discharge frequency changed during the period from the appearance of the target to the earliest EMG changes which occurred at about $80 \mathrm{msec}$ before movement). These cells are likely to participate in the generation of movement, and the changes in their discharge are not likely to be due to peripheral feedback. The results are shown in Figure 8, $A$ and $B$, for movements in directions at $45^{\circ}$ and $225^{\circ}$, respectively. It can be seen in Figure $8 A$ that, when movements at $45^{\circ}$ were made, most of the cells that were activated had a preferred direction at or within $\pm 45^{\circ}$ of the direction of movement. The distribution is symmetric around the direction of the upcoming movement. Conversely, when movements at $225^{\circ}$ were made (Fig. $8 B$ ), most of the cells that were inhibited had preferred directions at or within $\pm 45^{\circ}$ of the opposite direction of the movement. Therefore, a population with similar directional preferences was engaged early in both movements: in the first case, with an increase of activity; in the second, with a decrease in activity.

\section{Hislological analysis}

Of the 49 penetrations that were identified histologically, $15(30.6 \%)$ were in area $4,8(16.3 \%)$ were in the 4 / 6 border, and $26(53.1 \%)$ were in motor cortical area 6 which comprises the most posterior part of this area (Wiesendanger, 1981). Of 223 task-related cells recorded in these 49 penetrations, $80(36 \%)$ were in area 4,118 $(53 \%)$ were in area 6 , and $25(11 \%)$ were in the $4 / 6$ border. This border between area 4 and 6 was placed at the point of abrupt reduction in the number of large pyramidal cells in layer $V$, typical of area 4 . This border was more posterior at the mediolateral level in which we recorded as compared to more lateral or medial sites. This was due to the fact that very large pyramidal cells are more frequent in the part of area 4 controlling distal limb 


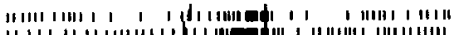

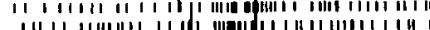

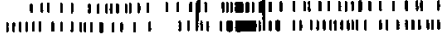

' ' '-5do' ' ' ' $d$ ' ' ' 'sdo' ' ' ioda
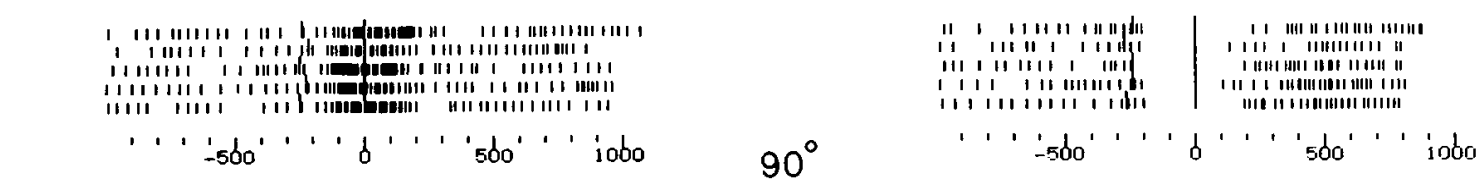

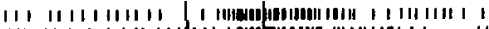

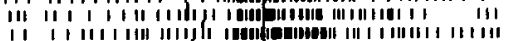

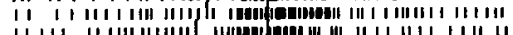

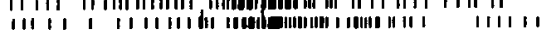
' ' ' -560 ' ' ' 1 ' ' ' 'sbo' ' ' 'iobo
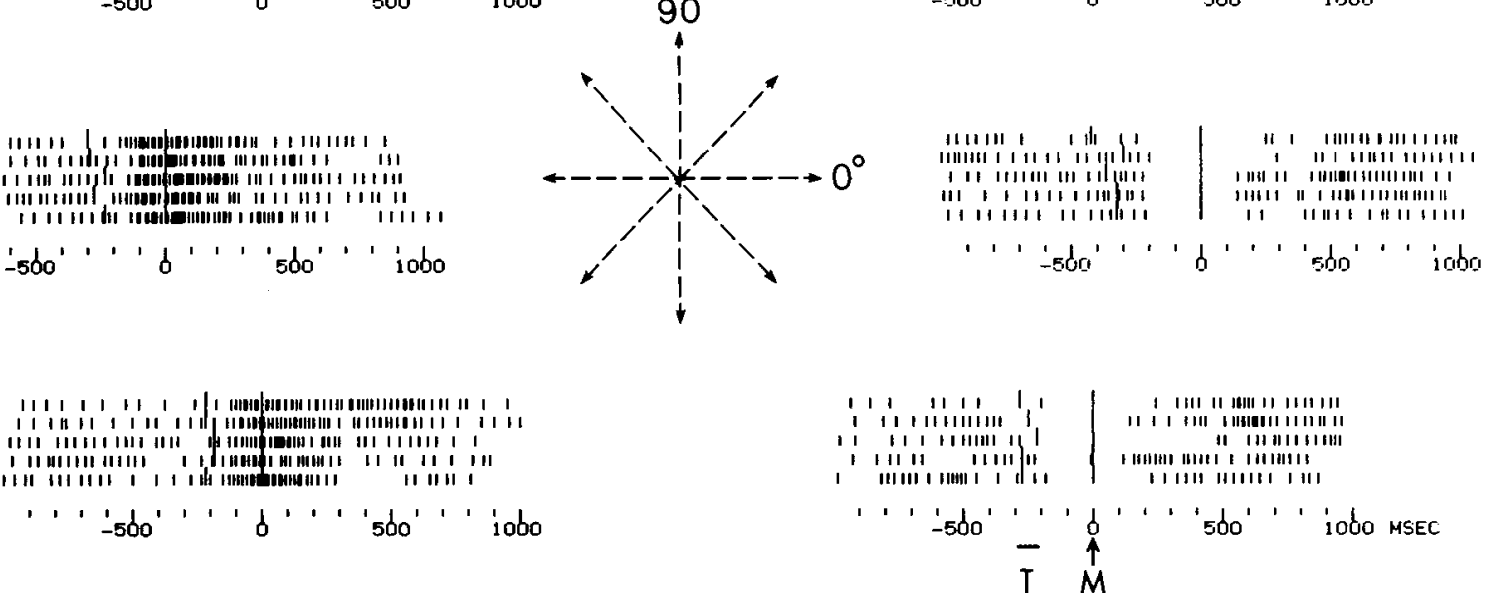

PCA1 10.501

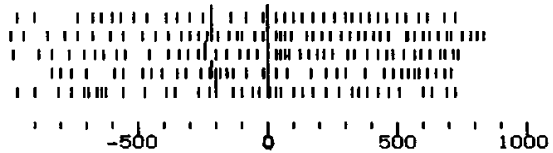

S1A

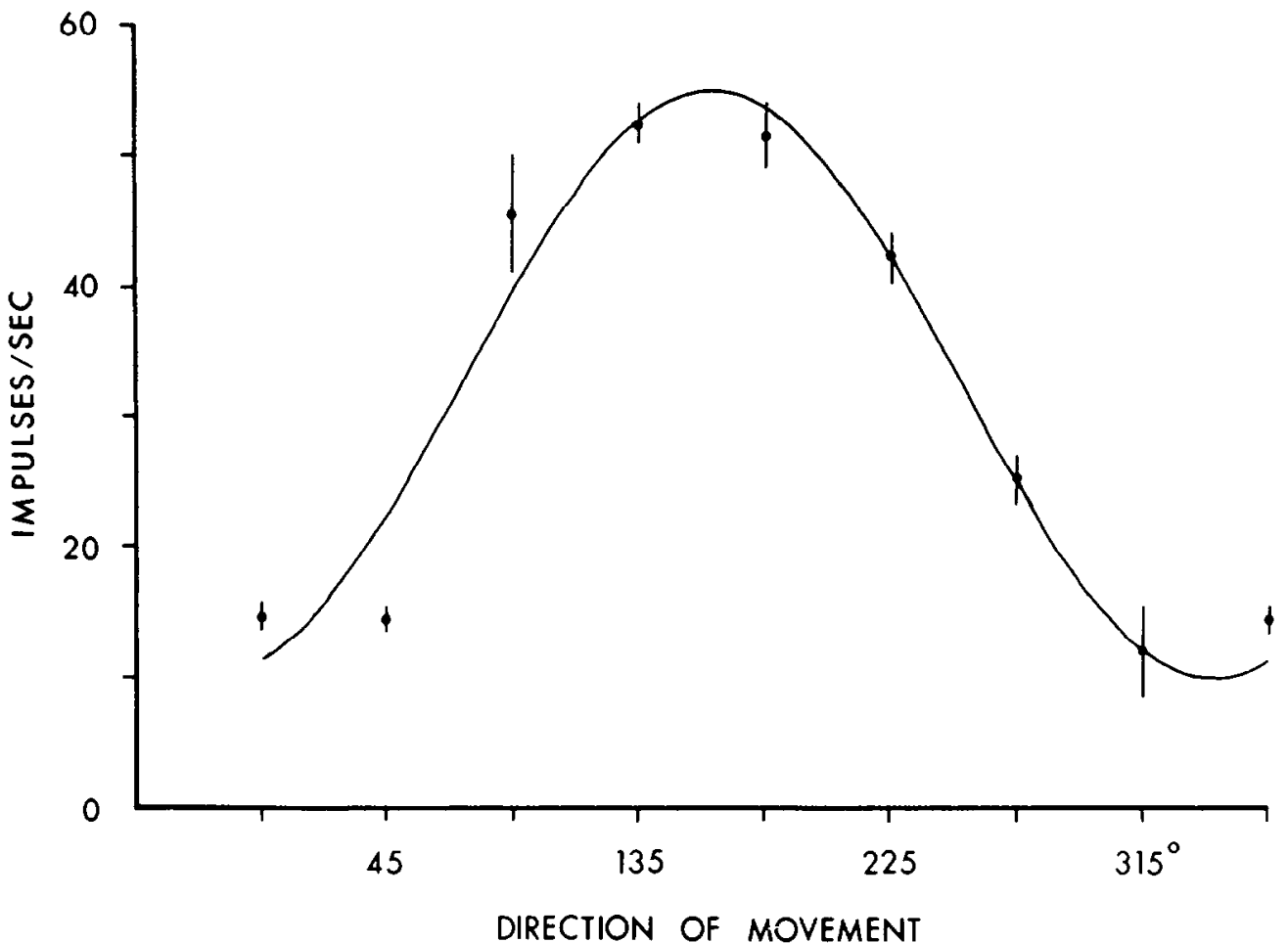

Figure 4. Orderly variation in the frequency of discharge of a motor cortical cell with the direction of movement. Upper half, Rasters are oriented to the movement onset, $M$, and show impulse activity during five repetitions of movements made in each of the eight directions indicated by the center diagram. Notice the orderly variation in cell's activity during the RT, MT, and TET. Lower half, Directional tuning curve of the same cell. The discharge frequency is for TET. The data points are the mean \pm SEM. The regression equation for the fitted sinusoidal curve is $D=32.37+7.281 \sin \theta-21.343 \cos \theta$, where $D$ is the frequency of discharge and $\theta$ is the direction of movement or, equivalently, $D=32.37+22.5 \cos \left(\theta-\theta_{0}\right)$, where $\theta_{0}$ is the preferred direction $\left(\theta_{0}=161^{\circ}\right)$. 


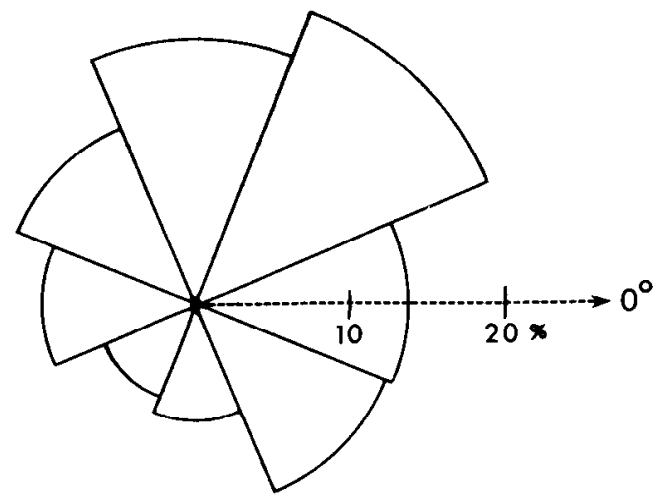

Figure 5. Frequency distribution (percentage) of the preferred directions of the 241 directionally tuned cells.

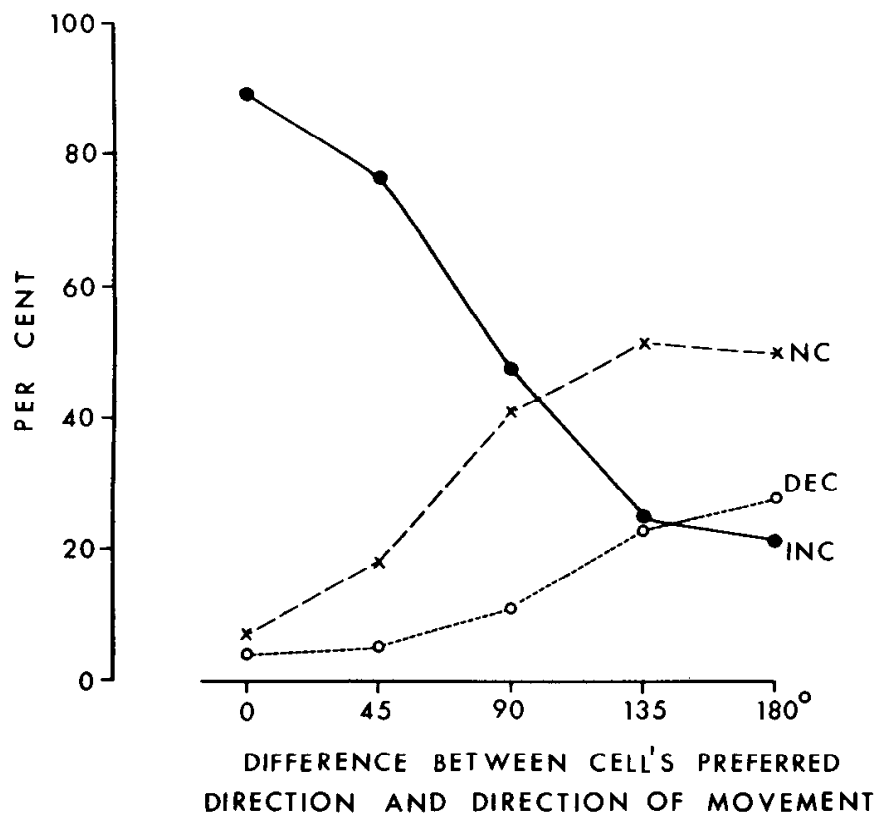

Figure 6. Relative frequency of occurrence of increase $(I N C)$, decrease $(D E C)$, and no change $(N C)$ in cell discharge as a function of the difference between cell's preferred direction and the direction of movement. The data were derived from the population of the 241 directionally tuned cells.

musculature (Wiesendanger, 1981). Using the abovon criteria for distinguishing areas 4 and $6,(a)$ no significant differences were observed in the directional properties of cells isolated in these cortical fields, $(b)$ cells with the same preferred direction were observed throughout the anteroposterior dimension of the cortex, and $(c)$ cells with similar preferred directions tended to cluster in depth. These questions are currently being investigated more thoroughly in our laboratory.

\section{Other observations}

Cells in the motor cortex did not discharge invariably with muscle contraction. The greatest increment in discharge usually occurred during voluntary limb movements aimed at securing a reward or when the animals manipulated objects or made small corrective movements to keep the manipulandum aligned to the target light. Yet, these same cells were frequently silent during other movements which also involved contraction of the same muscles. We did not investigate further this conditionality of activation.

Responses to passive manipulations were sought in every case. In general, cell discharge was much stronger during active movements than during passive manipulations. Of the latter, the most effective stimuli were rotations at joints and/or palpations of deep tissues. In several cases, responses to passive manipulations were complex in that some were evoked conditionally, depending on the animal making a movement, and others were depressed after a few stimuli but would reappear later on. Finally, in a few cases, presentation and/or movement of objects in the visual field evoked responses as described by Fetz et al. (1980). No attempt was made to investigate these responses further.

\section{Discussion}

We describe in this study the orderly relations between single cell activity in the motor cortex and the direction of two-dimensional arm movements. These relations are best described by a bell-shaped directional tuning curve: 241 of the 323 task-related cells (75\%) discharged at a higher frequency in relation to movements in a particular direction, and their activity decreased gradually with movement directions away from this preferred one. Other aspects of the changes in neuronal activity also varied with the direction of movement in an orderly fashion, including the sign of change (increase or decrease of discharge) and its time of onset. This variation of cell activity with the direction of movement could be the manifestation of a more fundamental relation between cell discharge and $(a)$ the direction of force exerted by the animal, $(b)$ the direction of the upcoming motion itself, or $(c)$ both of the above. Further experiments are needed to decide this question, since the direction of force and the direction of motion were partly confounded in the task used in the present study. It will be interesting to know, for example, whether the relations described above also are observed under isometric conditions (i.e., when forces of different directions are exerted isometrically); in that case, a generalization could be made of the force relation originally described by Evarts (1968) to include the direction as well as the magnitude of the force exerted by the animal. This problem is currently being investigated in our laboratory.

In general, the frequency of discharge was a sinusoidal function of the direction of movement. It is interesting that a similar relation has been described between the direction of eye movements and cell discharge in the paramedian pontine reticular formation (Henn and Cohen, 1976), the mesencephalic reticular formation (MRF) (Büttner et al., 1977), and the internal medullary lamina of the thalamus (Schlag and Schlag-Ney, 1977). Moreover, other aspects of cell activity in the MRF varied with the direction of eye movements in a manner similar to that observed in the present study; for example, cells tended to be activated earlier the closer their preferred direction was to that of the ensuing eye movement (Büttner et al., 1977). These observations suggest that the neural mechanisms controlling the direction of movement might be fundamentally similar in both the arm and the eye movement control systems.

The variation of cell discharge with the direction of 


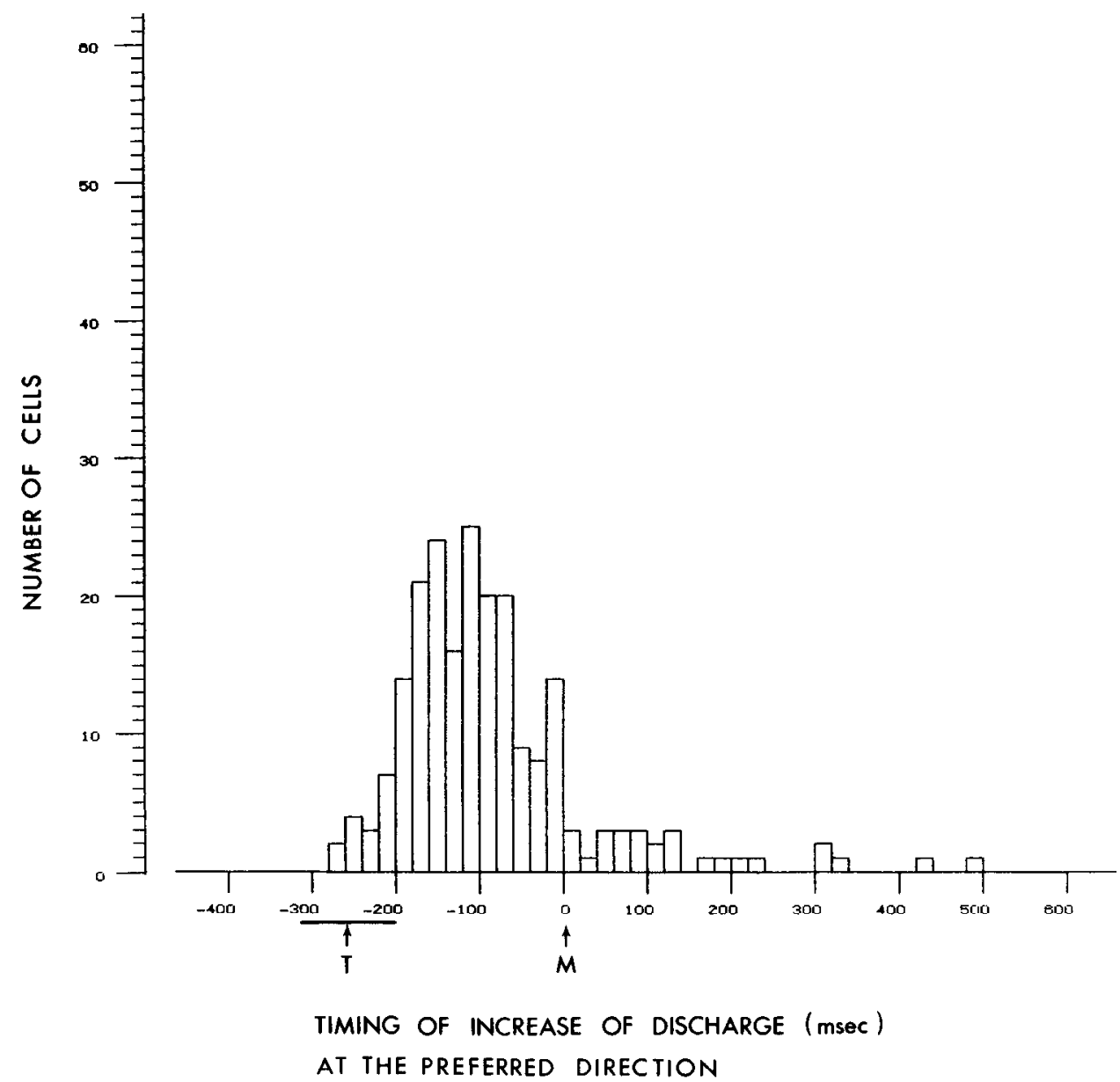

Figure 7. Distribution of the times of onset of the first increase in discharge when the direction of movement was within $\pm 22.5^{\circ}$ of the cell's preferred direction. $M$, Onset of movement; $T$, mean time \pm 1 SD of the appearance of the target.

A

MOVEMENT AT $45^{\circ}$

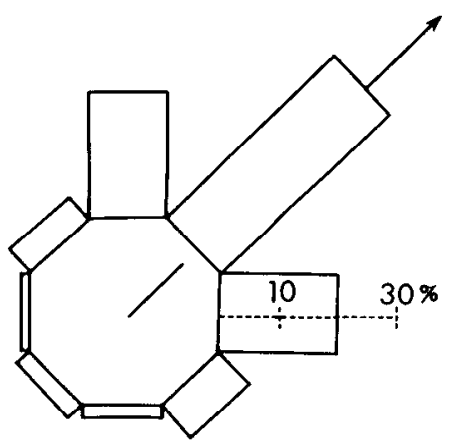

$B$

MOVEMENT AT $225^{\circ}$

Figure 8. Directional composition of "early" cells that changed activity during the period from the appearance of the target to the first changes in EMG activity $(80 \mathrm{msec}$ before movement onset). The solid $(A)$ and dashed $(B)$ bars refer to increases or decreases in activity, respectively. Notice that, for movements at $45^{\circ}$, this early "excited" population comprised cells with preferred directions within $\pm 45^{\circ}$ of the direction of the upcoming movement. Cells with such directional preferences were "inhibited" before movements in the opposite direction (at $225^{\circ}$ ). 
movement was observed in all epochs (RT, MT, and TET) and also before the onset of the earliest EMG changes. This and the fact that about $64 \%$ of the cells were activated before the earliest EMG changes and $87 \%$ before the onset of a movement in the cells' preferred direction (Fig. 7) suggest that the variation of cell discharge with movement direction is not the result of peripheral feedback. For the "earliest" cells, it probably reflects central input concerning the motor command for the direction of the upcoming movement. Of course, variation in cell discharge during the movement time might reflect peripheral feedback as well. It is noteworthy that the directional properties during the movement time were very similar to those in the reaction time. This may indicate a congruence in the spatial domain between the central command and peripheral input.

A salient finding of the present study was that a cell's discharge changed with several-not just one-movement directions; that is, individual cells did not "encode" movement direction in an absolute, one-to-one way. This indicates that movements in a particular direction are not subserved by motor cortical cells uniquely related to that movement. Instead, the orderly, sinusoidal variation in the tuning curve suggests that cells with overlapping tuning curves might cooperate to generate a movement trajectory in a desired direction. A hypothesis concerning this putative population code for movement direction was proposed recently (Georgopoulos et al., 1983).

When movements in a particular direction are to be made, the following changes seem likely to occur in motor cortical populations. Cells with preferred directions within $\pm 45^{\circ}$ of the direction of the ensuing movement will be activated shortly after the appearance of the target. These cells will discharge at various rates, depending on how close their preferred direction will be to that of the upcoming movement. At approximately the same time, a decrease in activity will occur in other cells with preferred directions away from that of the upcoming movement. The focus of activation and inhibition within the total population will change during movements in different directions and will partially overlap. The particular, for a given direction of movement, distribution of excitation and inhibition among directionally tuned cells would provide a population code for movement direction.

It is noteworthy that, in the task used in the present study, more cells were activated with movements directed away from than toward the body of the animal (Fig. 5). This probably can be explained by the fact that cells related to retraction movements were not active in the task (see "Results, Data base"). Given that the arm was already extended at the starting position, it seems likely that movements toward the body of the animal were assisted by gravity and were triggered by the inhibition of extension-related neurons (i.e., of cells with preferred directions pointing away from the animal). A different explanation is that, in the motor cortex, there may exist more cells related to extension than to flexion movements. Although proof of this hypothesis is lacking, a difference between some properties of corticomotoneuronal cells related to extension or flexion movements has been described; namely, the slopes of single cell activity versus force are twice as high for the former as for the latter (Cheney and Fetz, 1980). These workers proposed that "precentral cortex cells contribute a greater proportion of the descending input to extensor motoneurons" (Cheney and Fetz, 1980, p. 789). It should be mentioned that the trend shown in Figure 5 is not likely to be due to an unequal sampling of cortical populations, for the trend was present in each of the five hemispheres studied in spite of somewhat different locations of penetrations in each of these experiments.

The "matching process" hypothesis. The results of this study show that the motor cortex is engaged early ( $\sim 60$ to 80 msec after stimulus onsel) in the chain of events that are triggered by the appearance of the target and lead to the aimed movement. The avenues of this fast transmission of information to the motor cortex could involve corticocortical routes and/or subcortical structures, but the particular pathways involved in this transmission when aiming at targets remain to be determined.

We are confronted with a different problem when we try to understand how this visuospatial information is used for the generation of the aimed movement. According to a simple hypothesis, one could visualize this process as matching the requirements of the display to existing properties of motor cortical populations. For example, given two points on the plane, a straight line trajectory between the two is uniquely defined and its direction is fixed when one of the two points is assigned to be the starting point of the movement. Let us call it the "desired trajectory" (Td). Let us assume that there exist in the motor cortex populations of cells with spatial-directional properties such that, when these cells are activated, the hand moves through a certain trajectory; that is, to a certain direction within a certain part of space. Let us call it the "potential trajectory" (Tp). The hypothesized process would match Td with Tp: that is, it would select the appropriate response-related movement vector to match the stimulus-related visuospatial vector. Both vectors share the same "action space," but they are defined in different systems of coordinates; Td might be defined in terms of retinotopic, head-, or body-related coordinates; $\mathrm{Tp}$ might be defined in terms of muscle and joint coordinates. We make three postulates about this matching process. First, it is the outcome of learning. A similar process may be assumed to take place during the learning of visually guided reaching movements during the first months of life. Second, it effects transformations of information from one system of coordinates to another (e.g., from visual space to muscle and joint space). Third, it is widely distributed in that several neural structures participate in the stimulus-into-response spatial coding. It could be hypothesized that the gradual reduction in the variability of movement trajectories that occurs with motor learning (Georgopoulos et al., 1981) reflects a gradually increasing efficiency of this matching process.

Experimental evidence for this "matching process" is lacking, but the hypothesis poses requirements that can be tested. For example, a crucial part of it requires the presence of a mechanism in the motor cortex (or other motor structures) for coding the direction of movement independently of aiming at targets. Although this has not been investigated in complete darkness, available evidence indicates that such a mechanism does exist. For example, several studies have documented the differen- 
tial activation of motor cortical cells in relation to different directions of movements that are self-initiated (Fetz et al., 1980) or reactions (Evarts, 1968) but which do not involve aiming at targets. The most interesting part of the hypothesis is that relating to the transformation(s) of information from one system of coordinates to another. This is a central problem in understanding the visual guidance of motor behavior that awaits rigorous theoretical and experimental treatment.

\section{References}

Büttner, U., K. Hepp, and V. Henn (1977) Neurons in the rostral mesencephalic and paramedian pontine reticular formation generating fast eye movements. In Control of Gaze by Brain Stem Neurons, R. Baker and A. Berthoz, eds., pp. 309-318, Elsevier, Amsterdam.

Cheney, P. D., and E. E. Fetz (1980) Functional classes or primate corticomotoneuronal cells and their relation to active force. J. Neurophysiol. 44: 773-791.

Cochran, W. G., and G. M. Cox (1957) Experimental Designs, Ed. 2, John Wiley, New York.

Conrad, B., J. Meyer-Lohmann, K. Matsunami, and V. B. Brooks (1975) Precentral unit activity following torque pulse injections into elbow movements. Brain Res. 94: 219-236.

Evarts, E. V. (1966) Pyramidal tract activity associated with a conditioned hand movement in the monkey. J. Neurophysiol. 29: 1011-1027.

Evarts, E. V. (1968) Relation of pyramidal tract activity to force exerted during voluntary movement. J. Neurophysiol. 31: 1427.

Evarts, E. V. (1969) Activity of pyramidal tract neurons during postural fixation. J. Neurophysiol. 32: 375-385.

Evarts, E. V. (1981) Role of motor cortex in voluntary movements in primates. In Handbook of Physiology: The Nervous System, Sect. 1, Vol. II, Part 2, pp. 1083-1120, American Physiological Society, Bethesda, MD.

Evarts, E. V., and C. Fromm (1977) Sensory responses in motor cortex neurons during precise motor control. Neurosci. Lett. 5: 267-272.

Evarts, E. V., and J. Tanji (1976) Reflex and intended responses in motor cortex pyramidal tract neurons of monkey. J. Neurophysiol. 39: 1069-1080.

Fetz, E. E., D. V. Finocchio, M. A. Baker, and M. J. Soso (1980) Sensory and motor responses of precentral cortex cells during compatible passive and active joint movements. J. Neurophysiol. 43: 1070-1089.

Georgopoulos, A. P., J. F. Kalaska, and J. T. Massey (1980) Cortical mechanisms of two dimensional aiming arm movements. I. Aiming at different target locations. Soc. Neurosci. Abstr. 6: 156.

Georgopoulos, A. P., J. R. Kalaska, and J. T. Massey (1981) Spatial trajectories and reaction times of aimed movements: Effects of practice, uncertainty, and change in target location. J. Neurophysiol. 46: 725-743.

Georgopoulos, A. P., R. Caminiti, J. F. Kalaska, and J. T. Massey (1983) Spatial coding of movement: A hypothesis concerning the coding of movement direction by motor cortical populations. Exp. Brain Res. 7 (Suppl.), in press.

Hamada, I., and K. Kubota (1979) Monkey pyramidal tract neurons and changes of movement parameters in visual tracking. Brain Res. Bull. 4: 249-257.
Henn, V., and B. Cohen (1976) Coding of information about rapid eye movements in the pontine reticular formation of alert monkeys. Brain Res. 108: 307-325.

Hepp-Reymond, M. C., U. R. Wyss, and R. Anner (1978) Neuronal coding of static force in the primate motor cortex. J. Physiol. (Paris) 74: 287-291.

Howell, A. B., and W. L. Straus (1933) The Inuscular system. In The Anatomy of the Rhesus Monkey, E. G. Hartman and W. L. Straus, eds., pp. 89-175, Hafner; New York.

Humphrey, D. R., E. M. Schmidt, and W. D. Thompson (1970) Predicting measures of motor performance from multiple cortical spike trains. Science 179: 758-762.

Jasper, H. H. (1958) Recent advances in our understanding of ascending activities of the reticular system. In Reticular Formation of the Brain, H. H. Jasper, L. D. Proctor, R. S. Kringhton, W. C. Noshay, and R. T. Costello, eds., pp. 423434, Little, Brown and Co., Boston.

Lemon, R. N., and R. Porter (1976) Afferent input to movement-related precentral neurones in conscious monkeys. Proc. R. Soc. Lond. (Biol.) 194: 313-339.

Mardia, K. V. (1972) Statistics of Directional Data, Academic Press, New York.

Massey, J. T., R. Caminiti, J. F. Kalaska, and A. P. Georgopoulos (1981) Cortical mechanisms of two-dimensional aimed arm movements. VI. Electromyographic analysis. Soc. Neurosci. Abstr. 7: 563 .

Mountcastle, V. B., W. H. Talbot, H. Sakata, and H. Hyvarinen (1969) Cortical neuronal mechanisms in flutter-vibration studied in unanesthetized monkeys. Neuronal periodicity and frequency discrimination. J. Neurophysiol. 32: 452-484.

Mountcastle, V. B., J. C. Lynch, A. P. Georgopoulos, H. Sakata, and C. Acuna (1975) Posterior parietal association cortex of the monkey: Command functions for operations within extrapersonal space. J. Neurophysiol. 38: 871-908.

Schlag, J., and M. Schlag-Ney (1977) Visuonotor properties of cells in cat thalamic internal medullary lamina. In Control of Gaze by Brain Stem Neurons, R. Baker and A. Berthoz, eds., pp. 453-462, Elsevier, Amsterdam.

Schmidt, E. M., R. G. Jost, and K. K. Davis (1975) Reexamination of the force relationship of cortical cell discharge patterns with conditioned wrist movements. Brain Res. 83: 213-223.

Snedecor, G. W., and W. G. Cochran (1967) Statistical Methods, Ed. 6, Iowa State University Press, Ames, IA.

Thach, W. T. (1978) Correlation of neural discharge with pattern and force of muscular activity, joint position, and direction of intended next movement in motor cortex and cerebellum. J. Neurophysiol. 41: 654-676.

Wiesendanger, M. (1981) Organization of secondary motor areas of cerebral cortex. In Handbook of Physiology: The Nervous System, Sect. 1, Vol. II, Part 2, pp. 1121-1148, American Physiological Society, Bethesda, MD.

Wolpaw, J. R. (1980) Correlation between task-related activity and responses to perturbation in primate sensory motor cortex. J. Neurophysiol. 44: 1122-1137.

Wong, Y. C., H. C. Kwan, W. A. Mackay, and J. T. Murphy (1978) Spatial organization of precentral cortex in awake primates. I. Somatosensory inputs. J. Neurophysiol. 41: 11071119.

Woolsey, C. N., P. H. Settlage, D. R. Meyer, W. Spencer, P. Hamuy, and A. M. Travis (1950) Patterns of localization in the precentral and "supplementary" motor areas and their relation to the concept of a premotor area. Res. Publ. Assoc. Res. Nerv. Ment. Dis. 30: 238-264. 